\title{
Research on Computer Control Technology in the Background of Internet
}

\author{
Haiying Dan ${ }^{1, a}$ \\ ${ }^{1}$ Shaanxi Institute of Technology, Xi'an, Shaanxi, 710300 \\ ${ }^{a}$ email,
}

Keywords: Internet Age; Computer; Control Technology

\begin{abstract}
With the development of mobile Internet technology, computer control technology for the further development and improvement of the foundation, in people's daily life and industrial production in all areas have been applied to the computer control technology to achieve automation control, and the development of the camera technology to promote the various fields to the direction of intelligent and information technology for the development and progress of the industry made a great contribution, thus promoting the modernization of the progress and improvement of society. In the context of the Internet era achieves the information sharing, but also for the development of computer control technology to provide the basis.
\end{abstract}

\section{Introduction}

The development of computer control technology has been going on for a long time, but because of the social environment and the level of science and technology constraints, in people's lives and various fields have not been widely used, and now in the information age, mobile Internet technology rapid development, so that computer control technology into the tens of thousands of households, and in industrial and agricultural production and related technology applications, and the computer technology, Internet technology and digital technology, in recent years, the rapid development of Automated control in industrial production is possible [1]. Such as in the chemical industry production process is mainly through the Ethernet to achieve information transmission, but also for computer automation control to provide the basis for computer control technology in the chemical industry in the application is mainly reflected in the operation, acquisition, integration, assessment and other production processes, and automatic control of industrial production equipment for the production of chemical products to provide quality assurance, but also improve the production efficiency of chemical enterprises, chemical companies to develop a stable and rapid development to provide protection. In the process of the development of Chinese current society, the application of computer control technology under the background of mobile Internet has a positive effect on the development of modern enterprise information. Therefore, this paper first analyzes the computer control technology, and elaborates and explains the development of the computer control technology in the background of the mobile Internet, and provides reference and reference for the development of related technology fields.

\section{Analysis of Computer Control Technology}

Computer control technology is based on the automatic control technology and electronic information technology and computer theory as the theoretical basis, and the control technology as its main technical support, combined with SCM technology, programmable control technology as the basis, in the social production activities to improve production automation, precision and information technology, so that access to information better performance, to achieve the operation of automated control technology [2]. Computer technology was first born in the third period of industrial revolution, and after a long period of time, computer control technology in the industrial production process has been widely used, and automatic control in the industrial field, the development of computer control technology which has a certain impact on the automated production of Chinese enterprises, but because of the control technology in the operation process 
has a certain degree of difficulty, the need for relevant professional computer personnel to operate, so the popularity has been limited. Enterprises in the production process to better use of computer control technology, we must strengthen the introduction of computer professionals and training to ensure the rational operation of production automation and management, so as to protect the quality of production and production efficiency, but also the development of enterprises has brought a positive impact.

Generally speaking, the computer control technology is also the industrial control computer. From the macroscopic point of view, the computer control technology is divided into the hardware part and the software part, in which the hardware part provides the material basis for the computer control system, including the system bus, host, communication equipment, external equipment, man-machine contact equipment and input and output channels, as well as in the field of production of measurement sensors, etc., which plays a key role in the host, mainly to the various systems issued a command, and control the object to be controlled and detection, but also the core of computer control technology [3]. The external equipment mainly includes a console, a printer, a keyboard, a computer display terminal, a disk drive, etc., when the computer and the outside world information exchange is mainly to rely on these devices to achieve, and the production object and the computer between the exchange of information mainly through the input and output devices to achieve, the input channel is mainly in the computer to accept the signal after it is converted to digital signals, the production object parameters control, and the output channel is the production object data and command control. Measurement sensor is a parameter measurement of a device and the implementing agencies are mainly in the computer control signal output after the corresponding control action to control the object in accordance with the relevant requirements to run, but also an important part of computer control technology [4].

The software part is also an important component of computer control technology, which is the soul of computer technology, which mainly includes application software and system software. The application software mainly refers to the control program, man-machine interface program, input and output program, display programs, public subroutines, databases, etc., and the system software is mainly worth assembling, high-level language, operating system, control language, communication software. And the computer program operation is mainly dependent on the system software to achieve, in the system software, the operating system also includes the monitoring program, management procedures and system programs and other subroutines; and development system also contains simulation programs, service procedures, language processing procedures and data management procedures, etc.; diagnostic system, including debugging procedures and fault diagnosis procedures [5]. The main purpose of the application software is based on user needs, to solve the practical problems to write the program, is a user-oriented procedures to ensure that the control object is running a normal control procedures, service procedures; and the main function of the database is right Data retrieval, management and archiving, software design is to establish a database, and the relevant parameters required to call, query, display and modify.

\section{Computer Control Technology in the Background of Internet}

Centralized Control - Distributed Control. Centralized control mainly refers to a control center in the external information collection and processing of all, and in accordance with the information control issues in the corresponding solutions and improve the program, so that the problem is resolved for enterprises in the production and operation the problem is solved efficiently, and the system control efficiency is improved [6]. Prior to the computer centralized control technology applications, mainly to input or output work as a centralized control of the main body, its work is centralized control, the control center as its main core, the information input or output, the work also includes information on the content acquisition, processing, operation, feedback and display, computer control technology needs to work according to the specific content of the work to work and centralized control, first according to the information standards developed by the center to carry out information screening, and control center as a centralized control of information center, but in the process of centralized control of information in the event of a mistake, it will bring a greater 
impact, so that the use of computer control technology performance loss, which is a major drawback of centralized control technology.

The distributed control technology is different from the centralized control technology, distributed control technology is mainly through advanced computer technology and communication technology to control, its technical characteristics are strong, with a broad space for development, while distributed control is also known as distributed control system, the communication network as its core hub to control the operation of multiple systems to achieve the overall control of the computer system, distributed control in the work of the main work is the control technology, display technology, communication technology and computer technology, etc. Multiple technologies follow the principle of centralized operation, decentralized control, highlighting the flexibility of distributed control [7]. In the distributed control is mainly by virtue of computer control technology to achieve the control role in the computer equipment to achieve a variety of signal centralized processing, and at the same time according to its different functions will be divided into the host computer and the next machine two control methods, including the upper Machine is mainly through the computer to control the signal transmitted by the hub, and the lower computer is mainly through the computer technology to control the field signal.

Closed System - The Center Line. Closed system mainly refers to the external information is closed, not with the external environment, nor by the external environment and the impact of factors, this system to a certain extent, will have constraints on computer control technology, this system is not compatible and the development of the system, in the closed system, the organization and openness of the system is difficult to achieve, the system information cannot be shared, nor can the organization information be fully utilized and updated in real time.

In order to effectively transmit the signals between the relevant intelligent instruments and equipment, the controller equipment and the execution equipment used in the industrial production process, the industrial field center line system is integrated into the industrial production process. The system is mainly to make the two data between the data points for effective transmission, and through the way to achieve the center line of information transmission and data transmission [8]. The site midline system for industrial manufacturing enterprises with good reliability and economic and practical, the computer control is also relatively simple. The automatic control of the data in the industrial field is mainly based on the center line of the field, the signal and its data processing, through a certain technology to deal with the data in a balanced and orderly state, to complete the data and information of the automation control. The essence of the field center control is based on the decentralized control to enhance and optimize the decentralized control becomes more concentrated, so as to implement a unified management, that is dangerous dispersion, control separation.

Site Control - Enterprise Automation Control. Field control requires managers to meet the production site to supervise and control the production process, its essence is manual control, is the industrial production site generated by the signal and its related data processing, in order to achieve automatic control. However, the application of computer automation control technology in this period has improved the control efficiency, but it has been constrained and restricted by the environment. It is difficult to introduce the computer at the production site. Therefore, it is necessary to optimize the field control so that the field controls technology more perfect.

Enterprises in this case the implementation of automated control, which is the enterprise on-site control mode to optimize the results obtained at this stage in the Internet technology to fully play its role in the production site to introduce Ethernet industrial protocol, through the open Network on the industrial production site for all-round, the whole process of control. Internet technology in the computer control technology in the application, so that the products produced by the adaptability and safety factor has been significantly improved. However, the current situation, the enterprise through the Ethernet to the industrial production site control process is easy to be affected by the stability of the network signal, due to the production site environment closed, the network signal is not stable, it is difficult to ensure that the system work signal always remain stable [9]. At the same time, due to the large amount of information generated in the industrial production process, the 
exchange of signals and data in the process of Ethernet need to exchange and exchange of large amounts of information, and the replacement of new information and old information requires the Internet during the operation to refresh the data, when the Internet signal is unstable, it will make the system information processing problems. In order to solve the problem of instability in the process of running Ethernet, it puts forward the information chemical plant, which mainly transfers the large amount of data in the system during the transportation process so that the information data transmitted, collected and processed are in A stable environment to go, in order to achieve the automatic control of information, so that the industrial production site automation control efficiency and control quality has been significantly improved.

\section{Conclusion}

The application of computer control technology in industrial production can effectively improve the level of automation in the field of industrial production, realize the automatic control of industrial production and industrial production equipment, reduce the labor cost in industrial production, reduce the quality of production and The production efficiency is improved, the precision of the produced products is higher, compared with the artificial control, the machine equipment control can reduce the error caused by the artificial control, also reduces the personnel casualties phenomenon. With the development of computer technology, the theory of automation and control technology is more perfect, in the future industrial production process is bound to be combined with computer technology and automation and control technology, with the support of Internet technology to achieve information sharing, and the various units of the information data for effective transmission, improve the quality of industrial production.

\section{Acknowledgements}

Fund Project: Provincial (Shaanxi Provincial Education Science Planning Leading Group Office) Research on the Mode and Practice of Transforming Scientific Research Achievements into Teaching Resources in Higher Vocational Colleges. Number: SGH16V040

\section{References}

[1] Li Daqing. Fault-tolerant strategy of embedded computer control system[J]. Electronic World, 2017, (11): 76.

[2] Jiao Qiang. Upgrade technology to strengthen the information management Shijiazhuang City, Hebei Province, the use of large data platform for all-round supervision of elevator safety[J]. China Quality Technical Supervision, 2017, (04): 49.

[3] Zhai Haiwei. Computer information management in network security application research[J]. China Management Information, 2016,19 (16): 159-160.

[4] Xu Bin. Based on the automatic control of electronic measurement technology development[J]. Electronic production, 2016, (08): 93.

[5] Ouyang Ruixue.Study on Fault Tolerant Strategy of Embedded Computer Control System[J]. Information Technology and Information Technology, 2015, (12): 113-115.

[6] Wu Jing. Exploring the fault-tolerant strategy of embedded computer control system [J] .New China Communications, 2015,17 (18): 57.

[7] DANG Jie. Internet-based computer control technology research [J / OL]. Electronic production, 2015, (05): 158. (2015-03-27)

[8] Mou Yanxia. Steady grasp the pulse of the times to create a brilliant - written in the electronic six built 50 anniversary [J]. Microcomputer and application, 2015, 34 (03): $2+4$. 
[9] Xiao Yongliang, Xu Gou. Cloud network operation mode and visual control technology innovation[J]. Huxiang Forum, 2012, 25 (02): 46-48 + 76. 